\title{
Sex differences in risk factors for subclinical hypothyroidism
}

\author{
Jeonghoon Ha, Jeongmin Lee, Kwanhoon Jo, Dong-Jun Lim, Moo II Kang, Bong Yun Cha and Min-Hee Kim \\ Division of Endocrinology and Metabolism, Department of Internal Medicine, College of Medicine, The Catholic University of Korea, Seoul, Korea \\ Correspondence should be addressed to M-H Kim: benedict@catholic.ac.kr
}

\begin{abstract}
Objective: To investigate the prevalence of subclinical hypothyroidism $(\mathrm{SCH})$ in Korean adults and identify the risk factors for the occurrence of $\mathrm{SCH}$ by sex.

Design and methods: This study used data from the Sixth Korea National Health and Nutrition Examination Survey (KNHANES VI), a cross-sectional, nationally representative survey, which comprises a health interview survey, a health examination survey and a nutrition survey. To examine $\mathrm{SCH}$, the reference range of thyroid-stimulating hormone (TSH) was defined using both the range provided by the test kit manufacturer (SCH-M) and a population-based range (SCH-P). We investigated the prevalence of $\mathrm{SCH}$ and its risk factors by sex using both reference ranges.

Results: The prevalence of $\mathrm{SCH}$ in Koreans according to $\mathrm{SCH}-\mathrm{M}(0.35-5.5 \mu \mathrm{IU} / \mathrm{mL})$ was $5.6 \%$, and $3.3 \%$ with $\mathrm{SCH}-\mathrm{P}(0.62-6.68 \mu \mathrm{IU} / \mathrm{mL})$. For men, smoking significantly reduced the incidence of $\mathrm{SCH}$, positive anti-thyroid peroxidase antibody (TPOAb) significantly increased the risk of $\mathrm{SCH}$, and in an adjusted model, the risk of $\mathrm{SCH}$ in all quartiles increased as the urine iodine creatinine ratio (UICR) quartile increased. For women, positive TPOAb was confirmed as a risk factor for $\mathrm{SCH}$, as was the highest UICR quartile. Furthermore, the odds ratio for $\mathrm{SCH}$ in urban vs rural residence was 1.78.

Conclusions: The prevalence rates of $\mathrm{SCH}$ were similar to those reported in the literature and previously known risk factors were confirmed using both TSH reference ranges. The notable findings from this study are that the increased risk of $\mathrm{SCH}$ with increased iodine intake was more marked in men than in women and that residential area may be a risk factor for $\mathrm{SCH}$ in women.
\end{abstract}

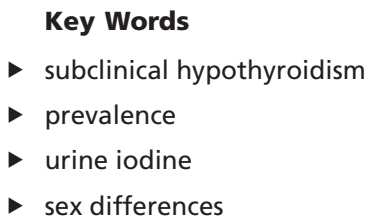

Endocrine Connections (2018) 7, 511-522

\section{Introduction}

Subclinical hypothyroidism (SCH) is defined as a mildly elevated serum thyroid-stimulating hormone (TSH) level with a serum free thyroxine (FT4) level within the normal range (1). While treatment of $\mathrm{SCH}$ is not mandatory in most cases, thyroid hormone replacement can be considered in pregnant patients who have TSH persistently $>10 \mu \mathrm{IU} / \mathrm{mL}$, associated symptoms or are at high risk of overt hypothyroidism $(2,3) . \mathrm{SCH}$ is frequently detected in the population with a prevalence of $3-12 \%(4,5)$. The prevalence is dependent on the TSH reference range used, and the TSH level is known to

$$
\begin{array}{lr}
\text { http://www.endocrineconnections.org } & \text { @ } 2018 \text { The authors } \\
\text { https://doi.org/10.1530/EC-18-0023 } & \text { Published by Bioscientifica Ltd }
\end{array}
$$

be strongly influenced by sex, area of residence, iodine intake and the presence of autoantibodies $(4,6,7)$. In iodine-rich areas, a prevalence of $\mathrm{SCH}$ of up to $18 \%$ has been reported $(2,7,8)$. Thyroid diseases including $\mathrm{SCH}$ are known to be more prevalent in women than in men $(9,10)$. Approximately $5-15 \%$ of women are positive for thyroid autoantibodies, either anti-thyroid peroxidase antibody (TPOAb) or anti-thyroglobulin antibody $(11,12)$. The higher incidence of thyroid autoantibodies in women is reported to account for their higher incidence of thyroid disease $(9,13)$. However, other sex-specific risk

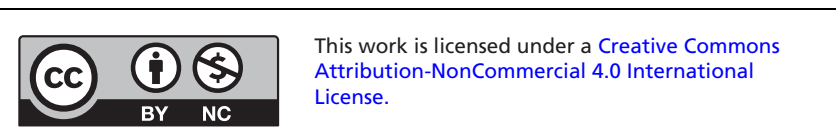


factors for SCH development have not been elucidated. In addition, the TSH reference range required to define the $\mathrm{SCH}$ varies depending on the target population, and the normal reference range varies between subgroups in the same population. For example, in a study of the Korean population, the prevalence of SCH varied from $0.16 \%$ to $17.63 \%$, depending on the particular population being studied $(2,14,15)$. In the present study, we investigated the prevalence of SCH in Korean adults based on data from the most recent nationally representative epidemiological survey, the Sixth Korea National Health and Nutrition Examination Survey (KNHANES VI) and examined the risk factors for the occurrence of SCH according to sex.

\section{Methods}

\section{Study population}

KNHANES VI was carried out by the Korea Centers for Disease Control and Prevention (CDC) and comprises a health interview survey, a health examination survey and a nutrition survey, using a stratified multistage probability sampling design. Informed written consent for participation was obtained from all study subjects. In addition, the study was approved by the Korea CDC Institutional Review Board. TSH reference range was calculated by two methods, and the subjects were divided into two groups according to the method used. First, we used the TSH reference range $(0.35-5.5 \mu \mathrm{IU} / \mathrm{mL})$ provided by the manufacturers of the test kit (Roche Diagnostics) to define SCH (SCH-M). Of the 22,948 Korean participants, 4914 aged $<19$ years were excluded. Subjects who were pregnant $(n=10)$, had delivered a child within the previous year $(n=62)$, had a history of treatment for thyroid disease $(n=58)$, for whom insufficient urine iodine data were available $(n=372)$, for whom insufficient FT4 data were available or were not within the normal range $(n=12,159)$ and for whom insufficient TSH data were available or were $<0.35 \mu \mathrm{IU} / \mathrm{mL}(n=69)$ were excluded from the analysis. Ultimately, 4888 participants were included in this study. Second, SCH was defined using a TSH reference range based on the enrolled population $(0.62-6.68 \mu \mathrm{IU} / \mathrm{mL})$ (SCH-P). Of the initial 22,948 Korean participants, 4801 subjects were selected for this analysis by excluding those who did not meet the inclusion criteria (subjects aged $<19$ years $(n=4914)$, pregnant $(n=10)$, history of delivery within 1 year $(n=59)$, history of treatment for thyroid disease $(n=57)$, insufficient urine iodine data $(n=368)$, insufficient FT4 data or not within the normal range $(n=12,643)$ and insufficient TSH data or value $<0.62 \mu \mathrm{IU} / \mathrm{mL}(n=167)$.

$$
\text { http://www.endocrineconnections.org }
$$

\section{Measurements}

All measurements and questionnaires were collected by specially trained investigators. Detailed anthropometric measurements were carried out as in previous studies $(16,17,18)$. Height and body weight were measured as part of the health examination. Body mass index (BMI) was calculated from the measured height and weight. BMI was categorized as underweight $\left(<18.5 \mathrm{~kg} / \mathrm{m}^{2}\right)$, normal weight $\left(\geq 18.5 \mathrm{~kg} / \mathrm{m}^{2},<23 \mathrm{~kg} / \mathrm{m}^{2}\right)$, overweight $\left(\geq 23 \mathrm{~kg} / \mathrm{m}^{2}\right.$, $\left.<25 \mathrm{~kg} / \mathrm{m}^{2}\right)$ or obese $\left(\geq 25 \mathrm{~kg} / \mathrm{m}^{2}\right)$. Waist circumference was measured to the nearest $0.1 \mathrm{~cm}$ at the narrowest point between the lowest rib and the uppermost lateral border of the right iliac crest. Blood pressure was also measured by trained technicians using a Baumanometer mercury sphygmomanometer (WA Baum, Copiague, NY, USA), and all the subjects were seated for at least $5 \mathrm{~min}$ before the measurements were taken. Creatinine, TSH, FT4 and TPOAb were measured from sampled blood collected after overnight fasting. TSH was measured using an E-TSH kit (Roche Diagnostics), for which the reference range was $0.35-5.50 \mu \mathrm{IU} / \mathrm{mL}$. FT4 was measured using an E-Free T4 kit (Roche Diagnostics) for which the reference range was $0.89-1.76 \mathrm{ng} / \mathrm{mL}$. TPOAb was measured using an E-Anti-TPO kit (Roche Diagnostics); the normal range for TPOAb in humans is $<34.0 \mathrm{IU} / \mathrm{mL}$. Serum creatinine was measured using a Hitachi Automatic Analyzer 7600-210 (Hitachi) and iodine intake status was evaluated using the urine iodine creatinine ratio (UICR) determined using inductively coupled plasma-mass spectroscopy (PerkinElmer). Socio-economic status was defined according to income categorized into quartiles. Chronic kidney disease (CKD) was divided into five stages based on the glomerular filtration rate by referring to the Kidney Disease Outcomes Quality Initiative classification (19).

\section{Definition of SCH}

SCH was defined as normal serum FT4 levels with serum TSH levels above the reference range. However, SCH-M $(0.35-5.5 \mu \mathrm{IU} / \mathrm{mL})$ differed from that defined using the 2.5-97.5 percentiles of serum TSH levels in the studied population (SCH-P, 0.62-6.68 $\mathrm{II} / \mathrm{mL}$ ). Although clinical practice guidelines suggest the use of population-based reference ranges for TSH (20), the upper limit of TSH $(6.68 \mu \mathrm{IU} / \mathrm{mL})$ acquired from the population data was higher than the cut-off values for SCH usually used in clinical practice. However, the clinical value of TSH upper limit values requires verification. Thus, we analyzed the data using both SCH-M and SCH-P.

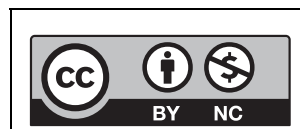

This work is licensed under a Creative Commons Attribution-NonCommercial 4.0 International License. 


\section{Statistical analysis}

Statistical analyses were performed using SAS, version 9.3 (SAS Institute, Cary, NC, USA). $P$ values $<0.05$ were considered significant. Data are expressed as numbers and percentages or as means \pm s.D. Differences between subjects with $\mathrm{SCH}$ and without SCH were evaluated using the Wilcoxon rank-sum test or the $\chi^{2}$ test, as appropriate. Differences between the four quartiles of serum TSH level were determined using a generalized linear model (Duncan's test of multiple comparisons). Multivariable adjusted logistic regression analysis was conducted to determine odds ratios (ORs) and 95\% confidence intervals (CIs) for the risk of SCH across variables. Because of the complex sampling design, a sampling weight method was used to assign participants representative of the Korean population for the analysis of data. Detailed characteristics of the statistical analysis of KNHANES have been reported previously $(16,21)$.

\section{Results}

\section{Baseline characteristics of the subjects}

\section{Using the manufacturer's suggested TSH reference range (SCH-M)}

The baseline characteristics of 4888 participants were analyzed, as were age, sex, BMI, region of residence, family history of thyroid disease, smoking status, UICR quartile,

Table 1 Baseline characteristics of subjects when kit manufacturer suggested TSH reference range $(0.35-5.5 \mu \mathrm{IU} / \mathrm{mL})$ is used.

\begin{tabular}{|c|c|c|c|c|}
\hline & \multirow[b]{2}{*}{ Total subjects $(n=4888)$} & \multicolumn{2}{|c|}{ Subclinical hypothyroidism } & \multirow[b]{2}{*}{$P$ value } \\
\hline & & No $(n=4597)$ & Yes $(n=291)$ & \\
\hline Age (years) & & & & 0.064 \\
\hline $19-29$ & $998(20.4 \%)$ & $944(20.5 \%)$ & $54(18.6 \%)$ & \\
\hline $30-39$ & $897(18.4 \%)$ & $855(18.6 \%)$ & $42(14.4 \%)$ & \\
\hline $40-49$ & $956(19.6 \%)$ & $903(19.6 \%)$ & $53(18.2 \%)$ & \\
\hline $50-59$ & $1016(20.7 \%)$ & $935(20.4 \%)$ & $81(27.8 \%)$ & \\
\hline $60-69$ & $927(19.0 \%)$ & $877(19.1 \%)$ & $50(17.2 \%)$ & \\
\hline 70 and above & $94(1.9 \%)$ & $83(1.8 \%)$ & $11(3.8 \%)$ & \\
\hline Sex & & & & $<0.001$ \\
\hline Male & $2545(53.9 \%)$ & $2434(54.8 \%)$ & $111(39.7 \%)$ & \\
\hline Female & $2343(46.1 \%)$ & $2163(45.2 \%)$ & $180(60.3 \%)$ & \\
\hline BMI $\left(\mathrm{kg} / \mathrm{m}^{2}\right)$ & & & & 0.436 \\
\hline$<18.5$ & $210(4.0 \%)$ & $197(4.1 \%)$ & $13(4.5 \%)$ & \\
\hline$\geq 18.5,<23$ & $1891(38.1 \%)$ & $1788(38.4 \%)$ & $103(35.4 \%)$ & \\
\hline$\geq 23,<25$ & $1168(24.4 \%)$ & $1096(24.3 \%)$ & $72(24.7 \%)$ & \\
\hline$\geq 25$ & $1619(33.5 \%)$ & $1516(33.2 \%)$ & $103(35.4 \%)$ & \\
\hline Region of residence ${ }^{a}$ & & & & 0.307 \\
\hline Rural & $1312(25.6 \%)$ & $1239(25.8 \%)$ & $73(22.8 \%)$ & \\
\hline Urban & $3576(74.4 \%)$ & $3358(74.2 \%)$ & $218(77.2 \%)$ & \\
\hline Family history of thyroid disease & & & & 0.787 \\
\hline No & $4644(95.0 \%)$ & $4366(95.0 \%)$ & $278(95.4 \%)$ & \\
\hline Yes & $244(5.0 \%)$ & $231(5.0 \%)$ & $13(4.6 \%)$ & \\
\hline Smoking & & & & $<0.001$ \\
\hline No & $2679(54.3 \%)$ & $2477(53.4 \%)$ & $202(70.4 \%)$ & \\
\hline Yes & $2209(46.7 \%)$ & $2120(46.6 \%)$ & $89(29.6 \%)$ & \\
\hline $\mathrm{UICR}^{\mathrm{b}}$ & & & & $<0.001$ \\
\hline Quartile 1 & $1246(25.1 \%)$ & $1207(25.8 \%)$ & $39(13.0 \%)$ & \\
\hline Quartile 2 & $1216(25.0 \%)$ & $1159(25.3 \%)$ & $57(21.1 \%)$ & \\
\hline Quartile 3 & $1198(24.8 \%)$ & $1122(24.7 \%)$ & $76(26.6 \%)$ & \\
\hline Quartile 4 & $1228(25.1 \%)$ & $1109(24.2 \%)$ & $119(39.3 \%)$ & \\
\hline Anti-TPO Ab & & & & $<0.001$ \\
\hline Absence & $4556(93.5 \%)$ & $4325(94.4 \%)$ & $231(78.0 \%)$ & \\
\hline Presence & $332(6.5 \%)$ & $272(5.6 \%)$ & $60(22.0 \%)$ & \\
\hline CKD stage & & & & 0.494 \\
\hline Stage 1, 2 & $4707(96.0 \%)$ & $4428(96.0 \%)$ & $279(96.8 \%)$ & \\
\hline Stage $3,4,5$ & $181(4.0 \%)$ & $169(4.0 \%)$ & $12(3.2 \%)$ & \\
\hline
\end{tabular}

*Data are presented as mean \pm S.D., $n$ (weighted \%), statistics were carried out using Rao-Scott Chi-square test; ${ }^{\mathrm{a}}$ classification of the area of residence is classified according to the administrative division of Korea; biodine intake status was evaluated by urine iodine creatinine ratio, quartile 1: <Q1 (141.53203438), quartile 2: $\geq$ Q1 and $<$ Q2 (281.81447737), quartile 3: $\geq$ Q2 and $<$ Q3 (664.64655625), quartile 4: $\geq$ Q3.

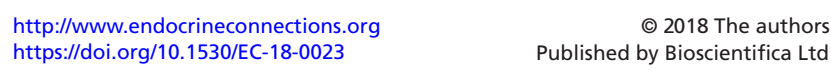


the presence of TPOAb and CKD staging (Table 1). The subjects were distributed evenly in age between 19 and 69 years, and only 94 subjects were aged over 70 years. There were 2343 (46.1\%) women, most of whom were urban area residents (74.4\%). Overall, 4644 (95.0\%) subjects had no family history of thyroid disease and 2679 (54.3\%) were non-smokers. UICR was uniformly distributed by quartile, and TPOAb was undetectable in 4556 (93.5\%) subjects. Most participants (96\%) were found to be in CKD stages 1 and 2 . The results of analysis of baseline characteristics by sex are summarized in Table 2 . There was a clear difference between men and women for smoking status: of the 2545 men, 1933 (76.0\%) were smokers, while only 276 of 2343 (11.8\%) women were smokers.

\section{Using the population-based TSH reference range (SCH-P)}

A total of 4801 subjects were analyzed for the same variables as shown in Table 1; 2303 (46.1\%) were women and most $(74.3 \%)$ were urban area residents. Details are shown in Table 2. UICR was uniformly distributed by quartile, and TPOAb was undetectable in 4472 (93.4\%) participants. As shown in Table 2 , there was a significant

Table 2 Association with subclinical hypothyroidism according to gender when kit manufacturer suggested TSH reference range is used.

\begin{tabular}{|c|c|c|c|}
\hline & \multirow{2}{*}{$\begin{array}{l}\text { Male total } \\
(n=2545)\end{array}$} & \multicolumn{2}{|c|}{$\begin{array}{l}\text { Subclinical hypothyroidism } \\
\text { (male) }\end{array}$} \\
\hline & & No $(n=2434)$ & Yes $(n=111)$ \\
\hline \multicolumn{4}{|l|}{ Age (years) } \\
\hline $19-29$ & $530(20.8 \%)$ & $504(20.7 \%)$ & $26(23.4 \%)$ \\
\hline 30-39 & $491(19.3 \%)$ & $472(19.4 \%)$ & $19(17.1 \%)$ \\
\hline $40-49$ & $502(19.7 \%)$ & $481(19.8 \%)$ & $21(18.9 \%)$ \\
\hline 50-59 & $502(19.7 \%)$ & $481(19.8 \%)$ & $21(18.9 \%)$ \\
\hline $60-69$ & $470(18.5 \%)$ & $451(18.5 \%)$ & $19(17.2 \%)$ \\
\hline $\begin{array}{l}70 \text { and } \\
\text { above }\end{array}$ & $50(2.0 \%)$ & $45(1.8 \%)$ & $5(4.5 \%)$ \\
\hline \multicolumn{4}{|l|}{ BMI $\left(\mathrm{kg} / \mathrm{m}^{2}\right)$} \\
\hline$<18.5$ & $65(2.6 \%)$ & $64(2.5 \%)$ & $1(0.9 \%)$ \\
\hline$\geq 18.5,<23$ & $837(32.9 \%)$ & $808(33.7 \%)$ & $29(26.2 \%)$ \\
\hline$\geq 23,<25$ & $659(25.9 \%)$ & $631(26.2 \%)$ & $28(25.2 \%)$ \\
\hline$\geq 25$ & $984(38.7 \%)$ & $931(37.6 \%)$ & $53(47.7 \%)$ \\
\hline \multicolumn{4}{|l|}{$\begin{array}{l}\text { Region of } \\
\text { residence }^{\mathrm{a}}\end{array}$} \\
\hline Rural & $693(27.2 \%)$ & $658(25.6 \%)$ & 35 (32.4\%) \\
\hline Urban & $1852(72.8 \%)$ & 1776 (74.4\%0 & $76(67.6 \%)$ \\
\hline \multicolumn{4}{|l|}{$\begin{array}{l}\text { Family history } \\
\text { of thyroid } \\
\text { disease }\end{array}$} \\
\hline No & $2442(96.0 \%)$ & $2334(95.7 \%)$ & $108(95.8 \%)$ \\
\hline Yes & $103(4.0 \%)$ & $100(4.3 \%)$ & $3(4.2 \%)$ \\
\hline \multicolumn{4}{|l|}{ Smoking } \\
\hline No & $612(20.1 \%)$ & $577(24.2 \%)$ & 35 (35.8\%) \\
\hline Yes & $1933(79.9 \%)$ & $1857(75.8 \%)$ & $76(64.2 \%)$ \\
\hline \multicolumn{4}{|l|}{$\mathrm{UICR}^{\mathrm{b}}$} \\
\hline Quartile 1 & $774(30.4 \%)$ & $760(30.5 \%)$ & $14(13.2 \%)$ \\
\hline Quartile 2 & $643(25.2 \%)$ & $617(25.1 \%)$ & $26(23.5 \%)$ \\
\hline Quartile 3 & $587(23.1 \%)$ & $556(23.8 \%)$ & $31(30.1 \%)$ \\
\hline Quartile 4 & $541(21.3 \%)$ & $501(20.6 \%)$ & $40(33.2 \%)$ \\
\hline \multicolumn{4}{|l|}{ Anti-TPO Ab } \\
\hline Absence & $2442(96.0 \%)$ & $2348(96.5 \%)$ & $94(84.7 \%)$ \\
\hline Presence & $103(4.0 \%)$ & $86(3.5 \%)$ & $17(15.3 \%)$ \\
\hline \multicolumn{4}{|l|}{ CKD stage } \\
\hline Stage 1,2 & $2445(96.0 \%)$ & $2338(96.1 \%)$ & $107(97.3 \%)$ \\
\hline Stage $3,4,5$ & $100(4.0 \%)$ & $96(3.9 \%)$ & $4(2.7 \%)$ \\
\hline
\end{tabular}

$\frac{P \text { value }}{0.696}$

Female total
$(n=2343)$
Subclinical hypothyroidism (female)

0.168

$\begin{array}{cll}145(6.2 \%) & 133(6.0 \%) & 12(4.8 \%) \\ 1054(50.0 \%) & 980(44.0 \%) & 74(38.9 \%) \\ 509(21.7 \%) & 465(22.1 \%) & 44(27.6 \%) \\ 635(27.1 \%) & 585(27.9 \%) & 50(28.7 \%)\end{array}$

0.161

No $(n=2163)$

Yes $(n=180)$

$440(20.3 \%) \quad 28(15.6 \%)$

$383(17.7 \%) \quad 23(12.8 \%)$

$422(19.5 \%) \quad 32(17.8 \%)$

$454(21.0 \%) \quad 60(33.3 \%)$

$426(19.7 \%) \quad 31(17.2 \%)$

$38(1.8 \%) \quad 6(3.3 \%)$

0.393

0.009

$\begin{array}{rrr}619(26.4 \%) & 581(26.1 \%) & 38(16.5 \%) \\ 1724(73.6 \%) & 1582(73.9 \%) & 142(83.5 \%)\end{array}$

0.981

0.596

$2202(94.0 \%) \quad 2032(94.1 \%) \quad 170(95.2 \%)$

$141(6.0 \%) \quad 131(5.9 \%) \quad 10(4.8 \%)$

0.020

$2067(88.2 \%) \quad 1900(88.7 \%) \quad 167(93.1 \%)$

$276(11.8 \%) \quad 263(11.3 \%) \quad 13(6.9 \%)$

0.001

$\begin{array}{lll}472(20.1 \%) & 447(20.0 \%) & 25(13.9 \%) \\ 573(24.5 \%) & 542(25.5 \%) & 31(17.2 \%) \\ 611(26.1 \%) & 566(25.9 \%) & 45(25.0 \%)\end{array}$

$79(43.9 \%)$

$<0.001$ $687(29.3 \%)$

$608(28.6 \%)$

$<0.001$

$2114(90.2 \%) \quad 1977(91.8 \%) \quad 137(73.6 \%)$

$229(9.8 \%) \quad 186(8.2 \%) \quad 43(26.4 \%)$

0.491

$2262(96.5 \%) \quad 2090(95.9 \%) \quad 172(96.5 \%)$

0.694

0.093

0.003

$81(3.5 \%) \quad 73(4.1 \%) \quad 8(3.5 \%)$

\begin{tabular}{c} 
P value \\
\hline 0.017
\end{tabular}

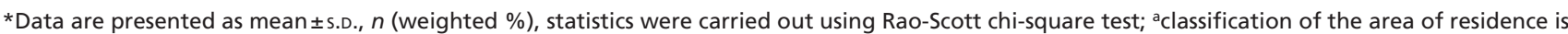
classified according to the administrative division of Korea; biodine intake status was evaluated by urine iodine creatinine ratio, quartile $1:<Q 1$ (141.53203438), quartile 2: $\geq Q 1$ and $<$ Q2 (281.81447737), quartile 3: $\geq$ Q2 and $<$ Q3 (664.64655625), quartile 4: $\geq$ Q3.

$$
\begin{array}{lr}
\text { http://www.endocrineconnections.org } & \text { @ } 2018 \text { The authors } \\
\text { https://doi.org/10.1530/EC-18-0023 } & \text { Published by Bioscientifica Ltd }
\end{array}
$$

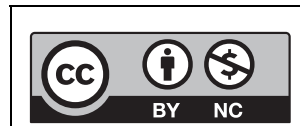

This work is licensed under a Creative Commons Attribution-NonCommercial 4.0 International License. 
difference between men and women in the frequency of smokers. The baseline values of other variables are similar to those shown in Table 1 and are summarized in Table 3. Table 4 shows the baseline characteristics analyzed by sex.

\section{Prevalence of $\mathrm{SCH}$ in the Korean population}

When SCH-M was used, the prevalence of SCH in Korea was 5.6\% (291 subjects) (Table 1) of whom 180 (60.3\%) were women. TPOAb was detected in 60 (22.0\%) subjects with SCH. There were 119 subjects in the highest quartile of UICR, accounting for $39.3 \%$ of all subjects with SCH. The prevalence of SCH differed significantly based on sex, the presence of TPOAb and UICR quartile $(P<0.001)$.
As shown in Table 2, the prevalence of SCH was 4.4\% (111 of 2545 ) in men and $7.7 \%$ (180 of 2343) in women.

When SCH-P was used, SCH was confirmed in 157 participants with a prevalence of 3.3\%. As with SCH-M, the occurrence of SCH significantly differed based on sex, presence of TPOAb and UICR quartile (Table 3).

In addition, there was a significant difference in the incidence of $\mathrm{SCH}$ according to smoking status regardless of the reference range used (Tables 1 and 3). However, when we analyzed male and female subjects separately using SCH-M, this difference was observed only in men (Table 2). As shown in Table 4, the prevalence of SCH using SCH-P was found to be $2.3 \%$ in men (58 of 2498 men) and 4.3\% in women (99 of 2303 women).

Table 3 Baseline characteristics of subjects when population-based TSH reference range $(0.62-6.68 \mu l \mathrm{U} / \mathrm{mL})$ is used.

\begin{tabular}{|c|c|c|c|c|}
\hline & \multirow[b]{2}{*}{ Total subjects $(n=4801)$} & \multicolumn{2}{|c|}{ Subclinical hypothyroidism } & \multirow[b]{2}{*}{$P$ value } \\
\hline & & No $(n=4644)$ & Yes $(n=157)$ & \\
\hline Age (years) & & & & 0.011 \\
\hline $19-29$ & $984(20.4 \%)$ & $957(20.6 \%)$ & $27(17.2 \%)$ & \\
\hline $30-39$ & $882(18.4 \%)$ & $863(18.5 \%)$ & $19(12.2 \%)$ & \\
\hline $40-49$ & $939(19.6 \%)$ & $904(19.5 \%)$ & $35(22.3 \%)$ & \\
\hline $50-59$ & $997(20.8 \%)$ & $950(20.5 \%)$ & $47(29.9 \%)$ & \\
\hline $60-69$ & $908(18.9 \%)$ & $883(19.0 \%)$ & $25(15.9 \%)$ & \\
\hline 70 and above & $91(1.9 \%)$ & $87(1.9 \%)$ & $4(2.5 \%)$ & \\
\hline Sex & & & & $<0.001$ \\
\hline Male & $2498(53.9 \%)$ & $2440(54.5 \%)$ & $58(36.4 \%)$ & \\
\hline Female & $2303(46.1 \%)$ & $2204(45.5 \%)$ & $99(63.6 \%)$ & \\
\hline BMI $\left(\mathrm{kg} / \mathrm{m}^{2}\right)$ & & & & 0.944 \\
\hline$<18.5$ & $207(4.0 \%)$ & $199(4.0 \%)$ & $8(4.2 \%)$ & \\
\hline$\geq 18.5,<23$ & $1859(38.3 \%)$ & $1795(38.2 \%)$ & $64(38.9 \%)$ & \\
\hline$\geq 23,<25$ & $1141(24.2 \%)$ & $1103(24.2 \%)$ & $38(25.7 \%)$ & \\
\hline$\geq 25$ & $1594(33.5 \%)$ & $1547(33.6 \%)$ & $47(31.2 \%)$ & \\
\hline Region of residence ${ }^{a}$ & & & & 0.076 \\
\hline Rural & $1293(25.7 \%)$ & $1258(25.9 \%)$ & $35(19.1 \%)$ & \\
\hline Urban & $3508(74.3 \%)$ & $3386(74.1 \%)$ & $122(80.9 \%)$ & \\
\hline Family history of thyroid disease & & & & 0.119 \\
\hline No & $4565(95.1 \%)$ & $4411(95.0 \%)$ & $154(98.0 \%)$ & \\
\hline Yes & $236(4.9 \%)$ & $233(5.0 \%)$ & $3(2.0 \%)$ & \\
\hline Smoking & & & & $<0.001$ \\
\hline No & $2641(54.5 \%)$ & $2534(54.0 \%)$ & $107(70.8 \%)$ & \\
\hline Yes & $2160(45.5 \%)$ & $2110(46.0 \%)$ & $50(29.2 \%)$ & \\
\hline UICR & & & & $<0.001$ \\
\hline Quartile 1 & $1226(25.1 \%)$ & $1204(25.5 \%)$ & $22(11.8 \%)$ & \\
\hline Quartile 2 & $1196(25.0 \%)$ & $1167(25.2 \%)$ & $29(21.9 \%)$ & \\
\hline Quartile 3 & $1179(24.9 \%)$ & $1140(25.0 \%)$ & $39(24.0 \%)$ & \\
\hline Quartile 4 & $1200(25.0 \%)$ & $1133(24.3 \%)$ & $67(42.3 \%)$ & \\
\hline Anti-TPO Ab & & & & $<0.001$ \\
\hline Absence & $4472(93.4 \%)$ & $4357(94.1 \%)$ & $115(72.2 \%)$ & \\
\hline Presence & $329(6.6 \%)$ & $287(5.9 \%)$ & $42(27.8 \%)$ & \\
\hline CKD stage & & & & 0.099 \\
\hline Stage 1, 2 & $4627(96.0 \%)$ & $4474(96.1 \%)$ & $153(98.3 \%)$ & \\
\hline Stage $3,4,5$ & $174(4.0 \%)$ & $170(3.9 \%)$ & $4(1.7 \%)$ & \\
\hline
\end{tabular}

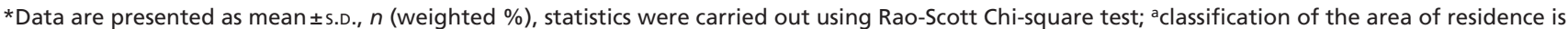
classified according to the administrative division of Korea; biodine intake status was evaluated by urine iodine creatinine ratio, quartile 1 : $<\mathrm{Q} 1$ (141.53203438), quartile 2: $\geq$ Q1 and $<$ Q2 (281.81447737), quartile 3: $\geq Q 2$ and $<$ Q3 (664.64655625), quartile 4: $\geq$ Q3.
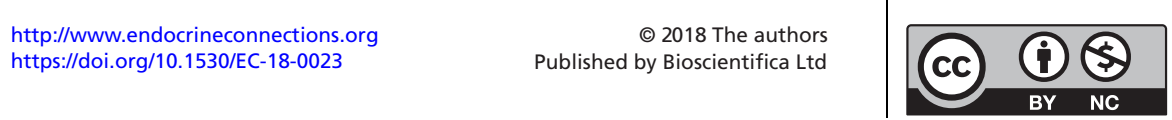

This work is licensed under a Creative Commons Attribution-NonCommercial 4.0 International License. 


\section{Risk factors associated with $\mathrm{SCH}$}

Tables 5 and 6 show the ORs for the occurrence of SCH for each variable according to the TSH reference range used and sex. Table 5 shows the relationship between different variables and the occurrence of SCH based on SCH-M. In the adjusted model, smoking significantly reduced the incidence of $\mathrm{SCH}$ in men (OR 0.58, CI: 0.34-1.00, $P=0.048)$. We also found that when TPOAb was positive, the occurrence of SCH was significantly increased in both crude (OR 5.03, CI: 2.68-9.45, $P<0.001$ ) and adjusted (OR 5.59, CI: 2.82-11.09, $P<0.001$ ) models. For urine iodine, the risk of SCH increased as the UICR quartile increased in the adjusted model (Table 5). For women, positivity for TPOAb was confirmed as a risk factor for $\mathrm{SCH}$, as was the highest iodine quartile (OR 2.41, CI: $1.44-4.05, P<0.001)$. In addition, the OR for SCH in urban vs rural residence was 1.78 (CI: 1.15-2.75, $P=0.010$ ). Similar results (summarized in Table 6) were obtained using SCH-P.

Table 4 Association with subclinical hypothyroidism according to gender when population-based TSH reference range is used.

\begin{tabular}{|c|c|c|c|c|c|c|c|c|}
\hline & \multirow{2}{*}{$\begin{array}{l}\text { Male total } \\
(n=2498)\end{array}$} & \multicolumn{2}{|c|}{$\begin{array}{c}\text { Subclinical hypothyroidism } \\
\text { (male) }\end{array}$} & \multirow[b]{2}{*}{$\boldsymbol{P}$ value } & \multirow{2}{*}{$\begin{array}{l}\text { Female total } \\
\qquad(n=2303)\end{array}$} & \multicolumn{2}{|c|}{$\begin{array}{l}\text { Subclinical hypothyroidism } \\
\text { (female) }\end{array}$} & \multirow[b]{2}{*}{$P$ value } \\
\hline & & No $(n=2440)$ & Yes $(n=58)$ & & & No $(n=2204)$ & Yes $(n=99)$ & \\
\hline Age (years) & & & & 0.852 & & & & 0.009 \\
\hline $19-29$ & $526(21.1 \%)$ & $513(21.1 \%)$ & $13(22.4 \%)$ & & $458(19.9 \%)$ & $444(20.1 \%)$ & $14(14.1 \%)$ & \\
\hline $30-39$ & $481(19.3 \%)$ & $472(19.4 \%)$ & $9(15.5 \%)$ & & $401(17.4 \%)$ & $391(17.7 \%)$ & $10(10.2 \%)$ & \\
\hline $40-49$ & $492(19.7 \%)$ & $479(19.6 \%)$ & $13(22.4 \%)$ & & $447(19.4 \%)$ & $425(19.3 \%)$ & $22(22.3 \%)$ & \\
\hline $50-59$ & $490(19.6 \%)$ & $477(19.5 \%)$ & $13(22.4 \%)$ & & $507(22.0 \%)$ & $473(21.5 \%)$ & $34(34.2 \%)$ & \\
\hline $60-69$ & $461(18.5 \%)$ & $452(18.5 \%)$ & $9(15.5 \%)$ & & $447(19.4 \%)$ & $431(19.6 \%)$ & $16(16.2 \%)$ & \\
\hline $\begin{array}{l}70 \text { and } \\
\text { above }\end{array}$ & $48(1.8 \%)$ & $47(1.9 \%)$ & $1(1.8 \%)$ & & $43(1.9 \%)$ & $40(1.8 \%)$ & $3(3.0 \%)$ & \\
\hline BMI $\left(\mathrm{kg} / \mathrm{m}^{2}\right)$ & & & & 0.999 & & & & 0.867 \\
\hline$<18.5$ & $63(2.5 \%)$ & $62(2.4 \%)$ & $1(2.5 \%)$ & & $144(6.3 \%)$ & $137(6.0 \%)$ & $7(5.2 \%)$ & \\
\hline$\geq 18.5,<23$ & $824(33.0 \%)$ & $804(33.5 \%)$ & $20(33.9 \%)$ & & $1035(44.9 \%)$ & $991(43.8 \%)$ & $44(41.7 \%)$ & \\
\hline$\geq 23,<25$ & $644(25.8 \%)$ & $629(26.0 \%)$ & $15(25.6 \%)$ & & $497(21.6 \%)$ & $474(22.0 \%)$ & $23(25.9 \%)$ & \\
\hline $\begin{array}{l}\text { Region of } \\
\text { residence }^{a}\end{array}$ & & & & 0.710 & & & & 0.009 \\
\hline Rural & $683(27.3 \%)$ & $666(25.9 \%)$ & $17(28.4 \%)$ & & $610(26.5 \%)$ & $592(26.0 \%)$ & $18(13.8 \%)$ & \\
\hline Urban & $1815(72.7 \%)$ & $1774(74.1 \%)$ & $41(71.6 \%)$ & & $1693(73.5 \%)$ & $1612(74.0 \%)$ & $81(86.2 \%)$ & \\
\hline $\begin{array}{l}\text { Family history } \\
\text { of thyroid } \\
\text { disease }\end{array}$ & & & & $\mathrm{N} / \mathrm{A}$ & & & & 0.297 \\
\hline No & $2398(96.0 \%)$ & $2340(95.7 \%)$ & $58(100.0 \%)$ & & $2167(94.1 \%)$ & $2071(94.1 \%)$ & $96(96.8 \%)$ & \\
\hline Yes & $100(4.0 \%)$ & $100(4.3 \%)$ & $0(0.0 \%)$ & & $136(5.9 \%)$ & $133(5.9 \%)$ & $3(3.2 \%)$ & \\
\hline Smoking & & & & 0.375 & & & & 0.147 \\
\hline No & $608(24.3 \%)$ & $592(24.9 \%)$ & $16(30.9 \%)$ & & $2033(88.3 \%)$ & $1942(88.8 \%)$ & $91(93.6 \%)$ & \\
\hline Yes & $1890(75.7 \%)$ & $1848(75.1 \%)$ & $42(69.1 \%)$ & & $270(11.7 \%)$ & $262(11.2 \%)$ & $8(6.4 \%)$ & \\
\hline UICR ${ }^{b}$ & & & & 0.003 & & & & 0.033 \\
\hline Quartile 1 & $763(30.5 \%)$ & $756(30.4 \%)$ & $7(11.2 \%)$ & & $463(20.1 \%)$ & $448(19.8 \%)$ & $15(12.1 \%)$ & \\
\hline Quartile 2 & $630(25.2 \%)$ & $617(25.0 \%)$ & $13(21.0 \%)$ & & $566(24.6 \%)$ & $550(25.4 \%)$ & $16(22.4 \%)$ & \\
\hline Anti-TPO Ab & & & & $<0.001$ & & & & $<0.001$ \\
\hline Absence & $2396(96.0 \%)$ & $2349(96.3 \%)$ & $47(82.6 \%)$ & & $2076(90.1 \%)$ & $2008(91.5 \%)$ & $68(66.2 \%)$ & \\
\hline Presence & $102(4.0 \%)$ & $91(3.7 \%)$ & $11(17.4 \%)$ & & $227(9.9 \%)$ & $196(8.5 \%)$ & $31(33.8 \%)$ & \\
\hline CKD stage & & & & 0.626 & & & & 0.071 \\
\hline Stage 1, 2 & $2404(96.2 \%)$ & $2348(96.3 \%)$ & $56(97.4 \%)$ & & $2223(96.5 \%)$ & $2126(95.8 \%)$ & $97(98.8 \%)$ & \\
\hline Stage $3,4,5$ & $94(3.8 \%)$ & $92(3.7 \%)$ & $2(2.6 \%)$ & & $80(3.5 \%)$ & $78(4.2 \%)$ & $2(1.2 \%)$ & \\
\hline
\end{tabular}

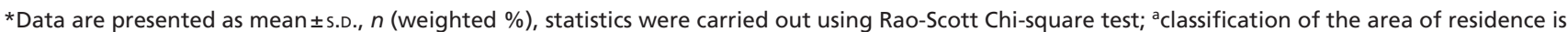
classified according to the administrative division of Korea; biodine intake status was evaluated by urine iodine creatinine ratio, quartile 1 : $<\mathrm{Q} 1$ (141.53203438), quartile 2: $\geq$ Q1 and $<$ Q2 (281.81447737), quartile 3: $\geq$ Q2 and $<$ Q3 (664.64655625), quartile 4: $\geq$ Q3. N/A, not available.

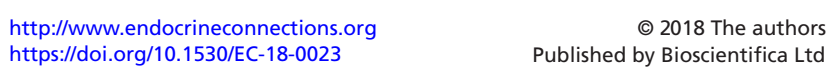


Table 5 Logistic regression analysis of subjects with subclinical hypothyroidism (kit manufacturer suggested TSH reference range is used).

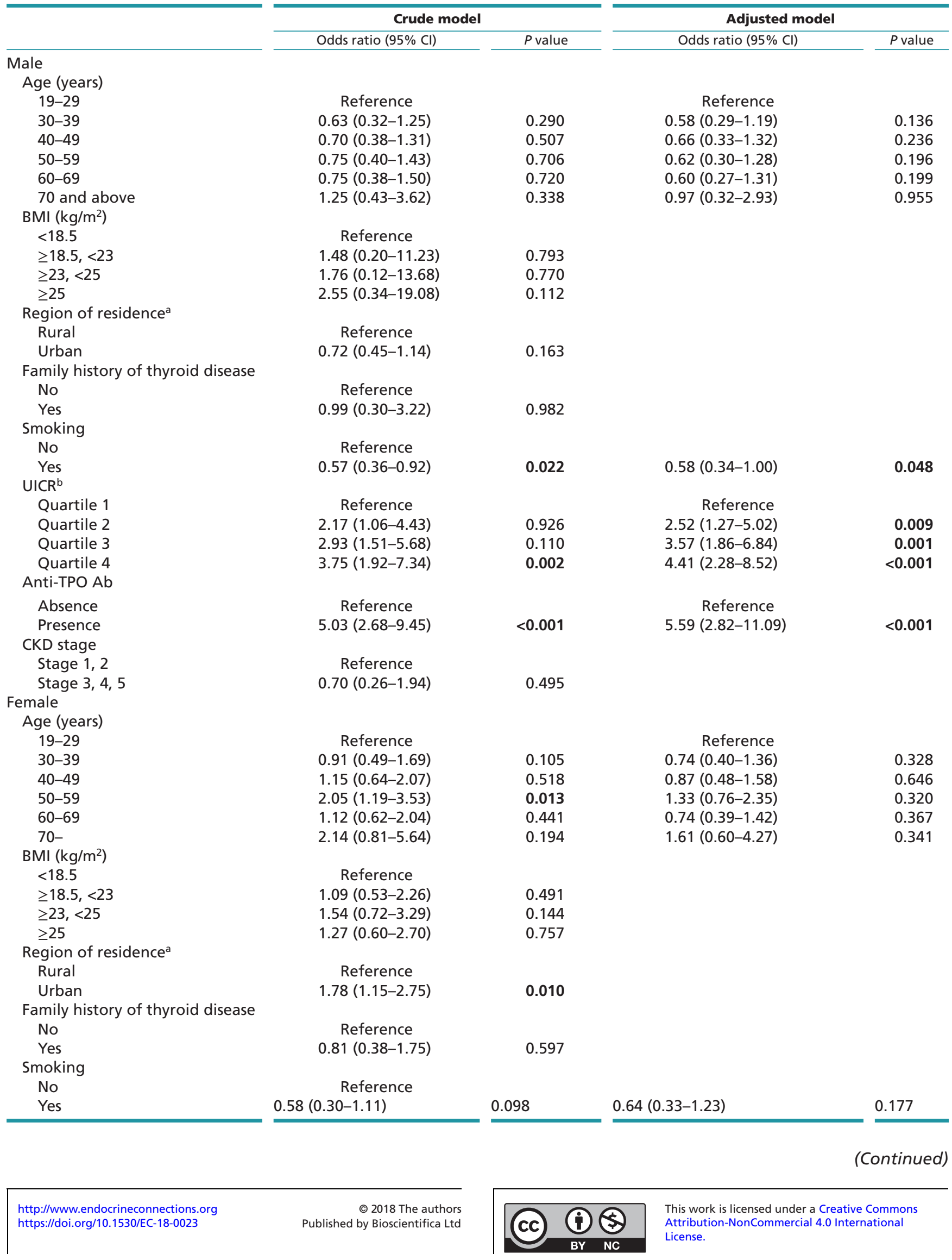


Table 5 Continued.

\begin{tabular}{|c|c|c|c|c|}
\hline & \multicolumn{2}{|c|}{ Crude model } & \multicolumn{2}{|c|}{ Adjusted model } \\
\hline & Odds ratio $(95 \% \mathrm{Cl})$ & $P$ value & Odds ratio $(95 \% \mathrm{Cl})$ & $P$ value \\
\hline \multicolumn{5}{|l|}{ UICR ${ }^{b}$} \\
\hline Quartile 1 & Reference & & Reference & \\
\hline Quartile 2 & $1.18(0.63-2.23)$ & 0.350 & $1.17(0.62-2.21)$ & 0.636 \\
\hline Quartile 3 & $1.45(0.83-2.54)$ & 0.873 & $1.46(0.82-2.60)$ & 0.203 \\
\hline Quartile 4 & $2.34(1.40-3.90)$ & $<0.001$ & $2.41(1.44-4.05)$ & $<0.001$ \\
\hline \multicolumn{5}{|l|}{ Anti-TPO Ab } \\
\hline Absence & Reference & & Reference & \\
\hline Presence & $4.00(2.59-6.16)$ & $<0.001$ & $4.06(2.63-6.28)$ & $<0.001$ \\
\hline \multicolumn{5}{|l|}{ CKD stage } \\
\hline Stage 1, 2 & Reference & & & \\
\hline Stage $3,4,5$ & $0.85(0.37-1.94)$ & 0.695 & & \\
\hline
\end{tabular}

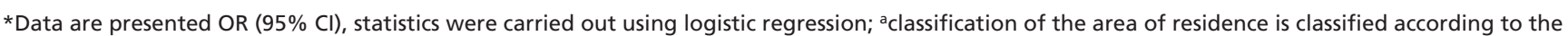
administrative division of Korea; biodine intake status was evaluated by urine iodine creatinine ratio, quartile 1: <Q1 (141.53203438), quartile 2: $\geq$ Q1 and $<$ Q2 (281.81447737), quartile 3: $\geq$ Q2 and $<$ Q3 (664.64655625), quartile 4: $\geq Q 3$.

\section{Discussion}

This study separately evaluated factors related to high prevalence of $\mathrm{SCH}$ in men and women to identify sexspecific risk factors for $\mathrm{SCH}$. In both sexes, regardless of whether SCH-P or SCH-M was applied, TPOAb positivity and high-iodine intake were related to the development of SCH. However, it was observed that the effect of iodine excess was more marked in men than in women. Urban residence was associated with $\mathrm{SCH}$ only in women.

Using SCH-M, the overall prevalence of $\mathrm{SCH}$ in Korea was $6.0 \%$ (4.4\% in men, $7.7 \%$ in women). Using SCH-P, the overall prevalence of $\mathrm{SCH}$ was $3.3 \%$ (2.3\% in men, $4.3 \%$ in women). The prevalence of SCH in Korea was reported previously to be $0.1-5.4 \%$ based on data from health care examinations $(15,22,23,24,25)$. However, these studies had limitations: their data were not representative of the whole population because the TSH cut-off levels used were different, and the results included only those subjects who underwent health care examinations. However, it is noteworthy that a similar prevalence was confirmed in our study using both a population-based TSH cut-off (SCH-P) and that suggested by the manufacturer of a commonly used kit (SCH-M).

Although the prevalence of $\mathrm{SCH}$ varies with geographical region, race, age, sex and TSH measurement method, most studies report that age, presence of autoantibody, female sex and iodine intake are risk factors for $\mathrm{SCH}$ (2). Most epidemiological studies have shown a higher prevalence of SCH in women than in men, but the underlying cause of this female predominance is unclear (2). The higher prevalence of autoantibodies is one possible cause. Pedersen et al. analyzed 4649 Danish subjects and reported that autoantibodies were more frequent in women (26). The prevalence of SCH was also associated with the presence of TPOAb. In the USA, National Health and Nutrition Examination Survey (NHANES) III reported a trend to higher prevalence of $\mathrm{SCH}$ in subjects who were positive for TPOAb (4). The Whickham survey reported similar results to those of NHANES III and showed that the ORs of developing overt hypothyroidism were four times higher in subjects with positive autoantibodies (27). Iodine intake is another risk factor for $\mathrm{SCH}$. Excessive iodine intake is known to cause thyroid dysfunction through direct toxic effects or immunological alterations (28). The prevalence of SCH has been shown to differ depending on whether it is reported from iodine-sufficient areas or from areas with an iodine deficit. According to the Laurberg study conducted in Northern Europe in 1998, the prevalence of SCH in Iceland, which is an iodinesufficient area, was $18 \%$, while that in the iodine-deficient area of Jutland, Denmark was 3.8\% (7). A study in China by Teng et al. reported that the prevalence of SCH differed between three regions according to their iodine intake, and a significant association between $\mathrm{SCH}$ and iodine intake was confirmed (29).

In our study, previously known risk factors for $\mathrm{SCH}$ were identified in both sexes regardless of the reference range used. The presence of TPOAb and the highest quartile of UICR increased ORs significantly in both men and women. Interestingly, we found that the change in risk of $\mathrm{SCH}$ according to the magnitude of iodine excess differed between sexes. Based on our results, men are more vulnerable to excessive iodine intake in terms of development of $\mathrm{SCH}$; a modest increase in iodine intake leads to a rapid increase in SCH risk. This finding was
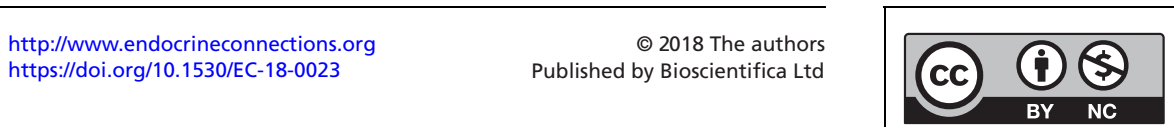

This work is licensed under a Creative Commons Attribution-NonCommercial 4.0 International License. 
Table 6 Logistic regression analysis of subjects with subclinical hypothyroidism (population-based TSH reference range is used).

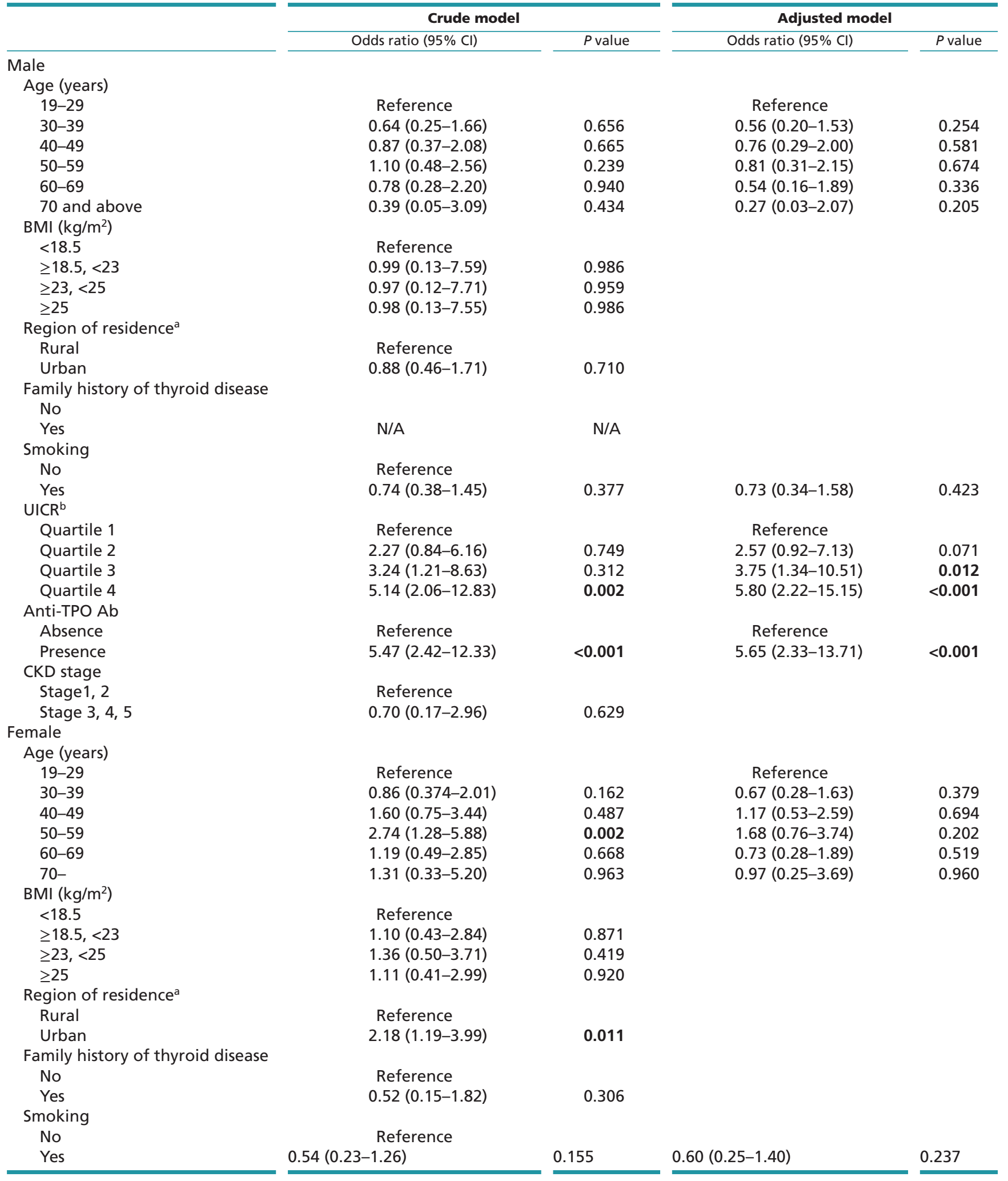


Table 6 Continued

\begin{tabular}{|c|c|c|c|c|}
\hline & \multicolumn{2}{|c|}{ Crude model } & \multicolumn{2}{|c|}{ Adjusted model } \\
\hline & Odds ratio $(95 \% \mathrm{Cl})$ & $P$ value & Odds ratio $(95 \% \mathrm{Cl})$ & $P$ value \\
\hline \multicolumn{5}{|l|}{$\mathrm{UICR}^{\mathrm{b}}$} \\
\hline Quartile 1 & Reference & & Reference & \\
\hline Quartile 2 & $1.45(0.63-3.37)$ & 0.921 & $1.40(0.59-3.29)$ & 0.445 \\
\hline Quartile 3 & $1.34(0.64-2.80)$ & 0.621 & $1.31(0.61-2.81)$ & 0.493 \\
\hline Quartile 4 & $2.52(1.30-4.88)$ & $<0.001$ & $2.67(1.35-5.26)$ & 0.005 \\
\hline \multicolumn{5}{|l|}{ Anti-TPO Ab } \\
\hline Absence & Reference & & erence & \\
\hline Presence & $5.49(3.26-9.22)$ & $<0.001$ & $5.33(3.19-8.90)$ & $<0.001$ \\
\hline \multicolumn{5}{|l|}{ CKD stage } \\
\hline Stage 1,2 & Reference & & & \\
\hline Stage $3,4,5$ & $0.28(0.06-1.24)$ & 0.093 & & \\
\hline
\end{tabular}

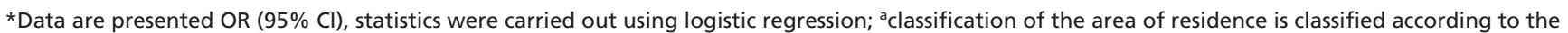

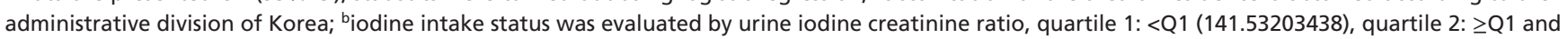
$<$ Q2 (281.81447737), quartile 3: $\geq Q 2$ and $<$ Q3 (664.64655625), quartile 4: $\geq Q 3$. N/A, not available.

consistent regardless of the TSH cut-off used. Using SCH$\mathrm{M}$, the ORs for SCH increased 2.5 times in the second quartile, 3.6 times in the third quartile and 4.4 times in the highest quartile relative to the lowest quartile (adjusted model shown in Table 5). When SCH-P was used, the ORs for SCH increased 2.6 times in the second quartile, 3.8 times in the third quartile and 5.8 times in the highest quartile (adjusted model shown in Table 6). In contrast, no sharp increase in ORs was observed in women as their UICR quartile increased. Even for those women in the highest quartile for UICR, the ORs did not exceed 3 (2.4 with SCH-M and 2.7 with SCH-P (Tables 5 and 6 , respectively).

Although the reason for this difference is not clear, there are several possible explanations. First, although the thyroid is the main reservoir for iodine intake, breast and cervical tissues are known to be involved in extrathyroidal iodine storage (30); thus, women are more capable of providing storage for excess iodine. Therefore, it may be that accumulation of excess iodine in the extrathyroidal tissues, which would attenuate iodine accumulation in the thyroid, may mitigate the adverse effects of excessive iodine intake on the development of $\mathrm{SCH}$. Second, estrogen has been demonstrated to have effects on thyroidal iodine uptake and TPO activity in animal models (31). Previous studies suggested that oestradiol might regulate iodide uptake via its action on the sodium/iodide symporter (NIS) and TPO, proteins that play important roles in thyroid hormone biosynthesis $(32,33,34)$. Oestradiol directly stimulates iodine uptake while regulating iodine uptake via reducing NIS or TPO gene expression (33). It is thought that oestradiol may regulate the effect of iodine on thyroid function. Because of the role of estrogen, the degree of response to iodine intake may differ in men and women. In other words, estrogen may modulate the effect of excessive iodine on thyroid tissue in women, which may reduce the risk of $\mathrm{SCH}$. However, considering that thyroid dysfunction frequently occurs in women, additional research is needed to evaluate other factors such as the concentrations of oestradiol and other co-factors.

Another distinctive finding of our study is the increased risk associated with the area of residence. In women, the risk of SCH was significantly higher in urban area residents than in rural area residents regardless of the TSH cut-off level used. The risk of SCH in urban residents was 1.78 times higher using SCH-M and 2.18 times higher using SCH-P (Tables 5 and 6, respectively). Although the underlying mechanism of this difference is unclear, it is possible that urban women have more opportunities to consume high-iodine meals or supplementation. However, a detailed survey to elucidate the lifestyle or food-intake patterns in terms of iodine exposure would be required to establish this.

Our study has several limitations. First, it is a crosssectional study and hence does not prove causality. Second, the presence of thyroid disease, dementia or other medical illness was self-reported. The use of medication and the detailed history of treatment were also assessed based on the information participants provided.

In conclusion, we examined the prevalence of $\mathrm{SCH}$ and the associated risk factors in the Korean population using the most recently released nationally representative data. The TSH reference ranges were calculated using two methods (the values proposed by the test kit manufacturer and population-based values) and the risk factors associated with SCH were analyzed. The prevalence rates
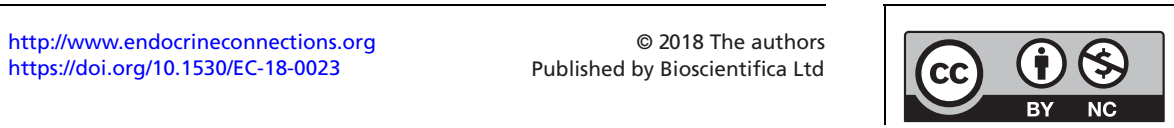

This work is licensed under a Creative Commons Attribution-NonCommercial 4.0 International License. 
of SCH were similar to those reported in the literature and previously known risk factors were confirmed using both TSH reference ranges. The notable findings of this study are that the increase in the risk of $\mathrm{SCH}$ with increasing iodine intake is more marked in men than in women and that residential area could be a risk factor for $\mathrm{SCH}$ in women. Further studies are needed to identify the mechanisms involved in these effects.

\section{Declaration of interest}

The authors report that they have no conflicts of interest that could be perceived as prejudicing the impartiality of the research reported.

\section{Funding}

The consultation for statistical analysis was supported by a grant from the Korea Health Technology R\&D Project through the Korea Health Industry Development Institute, funded by the Ministry of Health \& Welfare, Republic of Korea (Grant number: HI14C1062).

\section{References}

1 Fatourechi V. Subclinical hypothyroidism: an update for primary care physicians. In Mayo Clinic Proceedings, pp 65-71. Amsterdam, the Netherlands: Elsevier, 2009

2 Kim YA \& Park YJ. Prevalence and risk factors of subclinical thyroid disease. Endocrinology and Metabolism 201429 20-29. (https://doi. org/10.3803/EnM.2014.29.1.20)

3 Rosário PW, Carvalho M \& Calsolari MR. Natural history of subclinical hypothyroidism with $\mathrm{TSH} \leq 10 \mathrm{IU} / \mathrm{L}$ : a prospective study. Clinical Endocrinology 201684 878-881. (https://doi.org/10.1111/ cen.12939)

4 Hollowell J. T (4), and thyroid antibodies in the United States population (1988 to 1994): National Health and Nutrition Examination Survey (NHANES III). Journal of Clinical Endocrinology and Metabolism 200287 489-499. (https://doi.org/10.1210/ jcem.87.2.8182)

5 Kim WG, Kim WB, Woo G, Kim H, Cho Y, Kim TY, Kim SW, Shin MH, Park JW \& Park H-L. Thyroid stimulating hormone reference range and prevalence of thyroid dysfunction in the Korean population: Korea National Health and Nutrition Examination Survey 2013 to 2015. Endocrinology and Metabolism 201732 106-114. (https://doi. org/10.3803/EnM.2017.32.1.106)

6 Aghini-Lombardi F, Antonangeli L, Martino E, Vitti P, Maccherini D, Leoli F, Rago T, Grasso L, Valeriano R \& Balestrieri A. The spectrum of thyroid disorders in an iodine-deficient community: the Pescopagano survey. Journal of Clinical Endocrinology and Metabolism 199984 561-566.

7 Laurberg P, Pedersen KM, Hreidarsson A, Sigfusson N, Iversen E \& Knudsen PR. Iodine intake and the pattern of thyroid disorders: a comparative epidemiological study of thyroid abnormalities in the elderly in Iceland and in Jutland, Denmark. Journal of Clinical Endocrinology and Metabolism 199883 765-769. (https://doi. org/10.1210/jcem.83.3.4624)

8 Konno N, Makita H, Yuri K, Iizuka N \& Kawasaki K. Association between dietary iodine intake and prevalence of subclinical hypothyroidism in the coastal regions of Japan. Journal of Clinical Endocrinology and Metabolism 199478 393-397. (https://doi. org/10.1210/jcem.78.2.8106628)

9 Bjoro T, Holmen J, Kruger O, Midthjell K, Hunstad K, Schreiner T, Sandnes L \& Brochmann H. Prevalence of thyroid disease, thyroid dysfunction and thyroid peroxidase antibodies in a large, unselected population. The Health Study of Nord-Trondelag (HUNT). European Journal of Endocrinology 2000143 639-647. (https://doi.org/10.1530/ eje.0.1430639)

10 Tunbridge W, Evered D, Hall R, Appleton D, Brewis M, Clark F, Evans JG, Young E, Bird T \& Smith P. The spectrum of thyroid disease in a community: the Whickham survey. Clinical Endocrinology 19777 481-493. (https://doi.org/10.1111/j.1365-2265.1977.tb01340.x)

11 Cignini P, Cafà EV, Giorlandino C, Capriglione S, Spata A \& Dugo N. Thyroid physiology and common diseases in pregnancy: review of literature. Journal of Prenatal Medicine 2012664.

12 Kroopnick JM \& Kim CS. Overview of hypothyroidism in pregnancy. In Seminars in Reproductive Medicine. Stuttgart, Germany: Thieme Medical Publishers, 2016.

13 Strieder TG, Prummel MF, Tijssen JG, Endert E \& Wiersinga WM. Risk factors for and prevalence of thyroid disorders in a cross-sectional study among healthy female relatives of patients with autoimmune thyroid disease. Clinical Endocrinology 200359 396-401. (https://doi. org/10.1046/j.1365-2265.2003.01862.x)

14 Choi H, Park Y, Kim H, Choi S, Lim S, Park D, Jang H, Cho N \& Cho B. Prevalence of subclinical hypothyroidism in two population based-cohort: Ansung and KLoSHA cohort in Korea. Journal of Korean Thyroid Association 20103 32-40.

15 Chung JH, Kim BJ, Choi YH, Shin MH, Kim SH, Min YK, Lee MS, Lee MG \& Kim KW. Prevalence of thyrotoxicosis and hypothyroidism in the subjects for health check-up. Journal of Korean Society of Endocrinology 199914 301-313.

$16 \mathrm{Kim} \mathrm{J}$. Association between serum vitamin D, parathyroid hormone and metabolic syndrome in middle-aged and older Korean adults. European Journal of Clinical Nutrition 201569 425-430. (https://doi. org/10.1038/ejcn.2014.192)

17 Lee S, Han K, Yang H, Kim H, Cho J, Kwon H, Park Y, Cha B \& Yoon K. A novel criterion for identifying metabolically obese but normal weight individuals using the product of triglycerides and glucose. Nutrition and Diabetes 20155 e149. (https://doi.org/10.1038/ nutd.2014.46)

18 Song YS, Oh TJ, Kim KM, Moon JH, Choi SH, Jang HC, Park KS \& Lim S. Application of the 2013 American College of Cardiology/ American Heart Association Cholesterol Guideline to the Korean National Health and Nutrition Examination Surveys from 1998 to 2012. Diabetes and Metabolism Journal 201741 38-50. (https://doi. org/10.4093/dmj.2017.41.1.38)

19 Inker LA, Astor BC, Fox CH, Isakova T, Lash JP, Peralta CA, Tamura MK \& Feldman HI. KDOQI US commentary on the 2012 KDIGO clinical practice guideline for the evaluation and management of CKD. American Journal of Kidney Diseases 201463 713-735. (https://doi.org/10.1053/j.ajkd.2014.01.416)

20 Baloch Z, Guidelines Committee \& National Academy of Clinical Biochemistry. Laboratory medicine practice guidelines. Laboratory support for the diagnosis and monitoring of thyroid disease. Thyroid 200313 3-126. (https://doi.org/10.1089/105072503321086962)

21 Tran BT, Jeong BY \& Oh J-K. The prevalence trend of metabolic syndrome and its components and risk factors in Korean adults: results from the Korean National Health and Nutrition Examination Survey 2008-2013. BMC Public Health 201717 71. (https://doi. org/10.1186/s12889-016-3936-6)

22 Choi JK, Kim JS, Hwang CH, Kim DH, Hwang SW, Cho B \& Jul BY. Subclinical hypothyroidism and coronary risk factors in women above 55 years. Journal of the Korean Academy of Family Medicine 2002 23 96-103.

23 Jung CH, Sung KC, Shin HS, Rhee EJ, Lee WY, Kim BS, Kang JH, Kim H, Kim SW \& Lee MH. Thyroid dysfunction and their relation to cardiovascular risk factors such as lipid profile, hsCRP, and waist hip ratio in Korea. Korean Journal of Internal Medicine 200318146. (https://doi.org/10.3904/kjim.2003.18.3.146)

24 Lee WY, Oh KW, Rhee EJ, Jung CH, Kim SW, Yun EJ, Tae HJ, Baek KH, Kang MI \& Choi MG. Relationship between subclinical

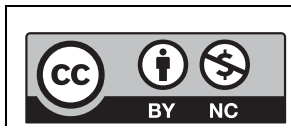

This work is licensed under a Creative Commons Attribution-NonCommercial 4.0 International License. 
thyroid dysfunction and femoral neck bone mineral density in women. Archives of Medical Research 200637 511-516. (https://doi. org/10.1016/j.arcmed.2005.09.009)

25 Oh MK, Cheon KS, Jung SM, Ryu DS, Park MS, Cheong SS, Kim JS $\&$ Park BG. Prevalence of thyroid diseases among adult for health check-up in a Youngdong area of Kwangwon province. Journal of the Korean Academy of Family Medicine 200122 1363-1374.

26 Pedersen IB, Knudsen N, Jørgensen T, Perrild H, Ovesen L \& Laurberg P. Thyroid peroxidase and thyroglobulin autoantibodies in a large survey of populations with mild and moderate iodine deficiency. Clinical Endocrinology 200358 36-42. (https://doi. org/10.1046/j.1365-2265.2003.01633.x)

27 Vanderpump M, Tunbrldge W, French J, Appleton D, Bates D, Clark F, Evans JG, Hasan D, Rodgers H \& Tunbridge F. The incidence of thyroid disorders in the community: a twenty-year follow-up of the Whickham Survey. Clinical Endocrinology 199543 55-68. (https://doi. org/10.1111/j.1365-2265.1995.tb01894.x)

28 Mahmoud I, Colin I, Many M-C \& Denef J-F. Direct toxic effect of Lodide in excess on Lodine-deficient thyroid glands: epithelial necrosis and inflammation associated with lipofuscin accumulation. Experimental and Molecular Pathology 198644 259-271. (https://doi. org/10.1016/0014-4800(86)90040-7)
29 Teng W, Shan Z, Teng X, Guan H, Li Y, Teng D, Jin Y, Yu X, Fan C \& Chong W. Effect of iodine intake on thyroid diseases in China. New England Journal of Medicine 2006354 2783-2793. (https://doi. org/10.1056/NEJMoa054022)

30 Ahad F \& Ganie SA. Iodine, iodine metabolism and iodine deficiency disorders revisited. Indian Journal of Endocrinology and Metabolism 20101413.

31 Lima LP, Barros IA, Lisbôa PC, Araújo RL, Silva AC, Rosenthal D, Ferreira AC \& Carvalho DP. Estrogen effects on thyroid iodide uptake and thyroperoxidase activity in normal and ovariectomized rats. Steroids 200671 653-659. (https://doi.org/10.1016/j.steroids.2006.03.007)

32 Boccabella AV \& Alger EA. Influence of estradiol on thyroid: serum radioiodide concentration ratios of gonadectomized and hypophysectomized rats. Endocrinology 196474 680-688. (https:// doi.org/10.1210/endo-74-5-680)

33 Canaris GJ, Manowitz NR, Mayor G \& Ridgway EC. The Colorado thyroid disease prevalence study. Archives of Internal Medicine 2000 160 526-534. (https://doi.org/10.1001/archinte.160.4.526)

34 Furlanetto TW, Nguyen LQ \& Jameson JL. Estradiol increases proliferation and down-regulates the sodium/iodide symporter gene in FRTL-5 cells. Endocrinology 1999140 5705-5711. (https://doi. org/10.1210/endo.140.12.7197)

Received in final form 1 March 2018

Accepted 7 March 2018

Accepted Preprint published online 7 March 2018 http://www.endocrineconnections.org https://doi.org/10.1530/EC-18-0023 (c) 2018 The authors Published by Bioscientifica Ltd

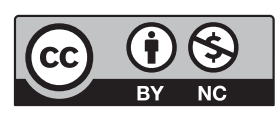

This work is licensed under a Creative Commons Attribution-NonCommercial 4.0 International License. 